\title{
CFD simulation of a CT scan oral-nasal extrathoracic model
}

\author{
C. Paz ${ }^{1}$, E. Suárez ${ }^{1}$, M. Concheiro ${ }^{1}$, J. Porteiro ${ }^{1}$ \& R. Valdés ${ }^{2}$ \\ ${ }^{1}$ Escuela de Ingeniería Industrial, University of Vigo, Spain \\ ${ }^{2}$ Otorhinolaryngol service, Povisa Hospital, Spain
}

\begin{abstract}
The study of air flow dynamics through the respiratory system is one of the leastresearched areas in the biofluid field. Computational fluid dynamics (CFD) has become an essential tool for analysing the airway flows due to the complex geometries involved. In this research a full process for obtaining the CFD simulation results from a CT scan has been developed. The complete extrathoracic oro-nasal airways, and the upper tracheobronchial region, have been studied in this research. The nasal route up to the trachea was obtained from CT images of a healthy woman, with the help of the free medical reconstruction software InVesalius. The oral cavity and lower airways were modelled from previous bibliographic geometry. The stereolithography file extracted from the scan was joined in a CAD program together with the other geometry and included the outer surface of a person and a far wall to simulate more realistic breathing conditions. The meshing process was performed with Gambit and Tgrid, and simulations were done with the Ansys solver Fluent. The velocity and pressure fields were calculated for the entire range of physiological capacity. The flow behaviour and respiratory effort under different breathing conditions were analysed. The volume airflow and pressure drop curves through oral and nasal routes were compared at difference activity levels. Interesting behaviours were found when comparing different breathing pattern results, and the importance of using a realistic geometry was demonstrated. This study has also provided the basis for the development of simulations starting from scanner data for future research. Some technical aspects in automated scan reconstruction still need to be resolved before this process become a standard tool for rhinology Keywords: oral-nasal, CFD, extrathoracic model, airways, far condition.
\end{abstract}




\section{Introduction}

The aerodynamics inside the airway is determined by the complex morphology and the flow rate. Computational fluid dynamics (CFD) techniques are already been used for some time to simulate flow behaviour in the human airway system $[1,2]$, given the impossibility of making direct measurements of flow patterns inside. CFD techniques are mostly used to simulate idealized and simplified airway models [3, 4]. Considerable differences were found when comparing results of CT based real models and simplified models. Xi and Longest [5] studying particle deposition in comparison to experimental data, concluded that the real geometry provides the best predictions of local particle deposition. Pharynx and larynx morphologic details should be considered as key parameters affecting the flow characteristics (Jayaraju et al. [6]).

Modelling realistic geometries is important to advance the understanding of the behaviour of breathing process. Truly realistic geometry models have recently been made available. In this paper a CT based realistic extrathoracic airway model has been simulated. The objective of this research is to analyse the breathing process in real extrathoracic airways. The important effect of breathing patterns (Saksono et al. [7]): nasal, oral, and simultaneous nasal and oral breathing has been analysed. The pressure jumps simulated correspond to flow rates from a state of rest (15 l/min) to extreme heavy breathing (>100 l/min). Improving CFD techniques with respect to these real airway geometries may improve knowledge of the breathing process. Medical practice could be improved in areas such as inhalation devices (Inthavong et al. [8]), obstructions, respiratory problems (Zhang and Kleinstreuer [9]), mechanical ventilation and help for respiratory system surgeons.

\section{Model generation}

The development of the CFD model began with the acquisition of the scanner images. The complete process is summarized in figure 1. Computed tomography (CT) is the most common technique used for examination of airways disease because it permits the visualization of the soft tissues. The CT is a consolidated technique, forty years old.

Basically it consists of the creation of a greyscale 2-D projection image of a 3-D object by passing electromagnetic radiation through the object of interest. The different tissues, thickness and density, attenuate the rays to different degrees, creating a high contrast image of the patient scanned. Each scan image consists of a large number of pixels, and each pixel is assigned a numerical value, grey scale corresponding witch the tissue. Series of slices were taken at sagittal, coronal and axial planes each $1.5 \mathrm{~mm}$. All these airway contiguous slices were stored as Digital Imaging and Communications in Medicine (DICOM) standard. The scans analysed in this research correspond to a healthy 30-year-old European Caucasian female. 

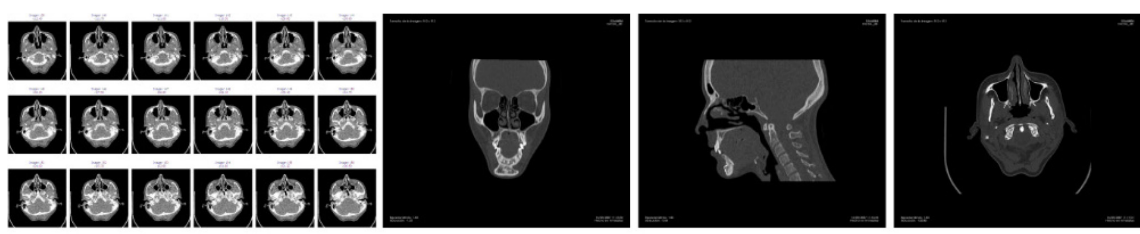

1-Detailed CT scan images, (DICOM format)

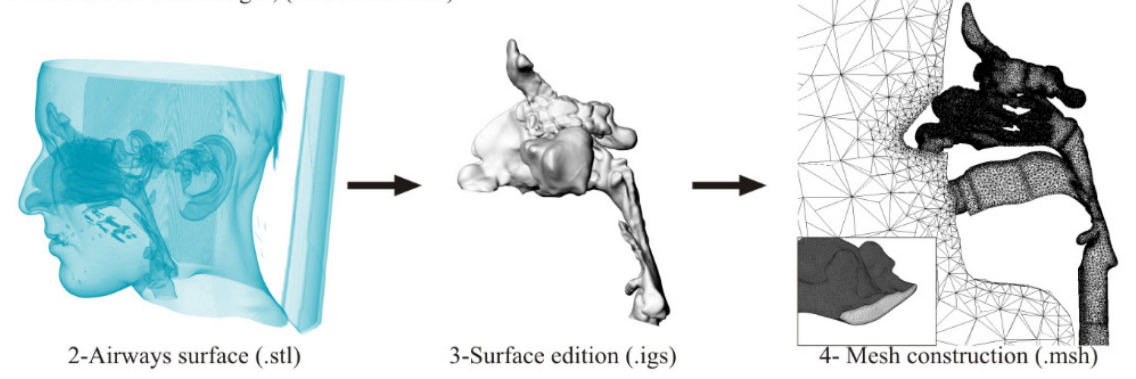

Figure 1: $\quad$ Procedure for creating the CFD model from CT scans.

\subsection{InVesalius}

Recent advances in image treatment and segmentation have made the recognition and reconstruction of human structures acquired using CT possible. The utilization of these reconstructions is a relatively new research technique. InVesalius is a free medical software, developed at CTI (Renato Archer Technology of Information Center), a research institute of Brazilian Science and Technology Center. InVesalius was used to generate the virtual threedimensional model corresponding to healthy patient airways. After a threedimensional reconstruction of .dcm images, the software was used to generate the .stl (stereolithography) files. The extraction of a 3D surface of the region to be studied was the first step; this process is called segmentation. In this process different filters and algorithms compare each pixel value with its neighbours and found similarities so that edges could be created. Each segmented region is defined according to the pixel value which can be equal to or higher than a defined threshold value. This process was carried out with the program defaults parameters set for airways. However the process could be adjusted manually with different filters and algorithms (Kiraly et al. [10] and Beare [11]). The edges created in each slide sweep was exported as a complete surface (.stl)

Sagittal, coronal and axial scans generate three different .stl files. Joining these files a point cloud was generated and taking the highest resolution image a unique geometry was obtained, as a NURBS approach (Piegl and Tiller [12]). The final igs file reconstructs the real geometry faithfully. 


\subsection{Complete model}

The full geometry model was generated in the NURBS modelling software Rhinoceros. The scan based reconstructed geometry was completed with other previously tested bibliographic models to complete a full extrathoracic airways model. The oral cavity of Johnstone et al. [13] was fused with the scanned geometry with a smooth transition. In a similar way the trachea used in the FSI model of Malvè et al. [14] was coupled to our geometry. To provide more realistic boundary conditions the facial skin obtained from the CT was used as the frontier of the domain, and the region studied included a big sphere with a far region, considered to be distant enough to maintain constant atmospheric pressure and null velocity.

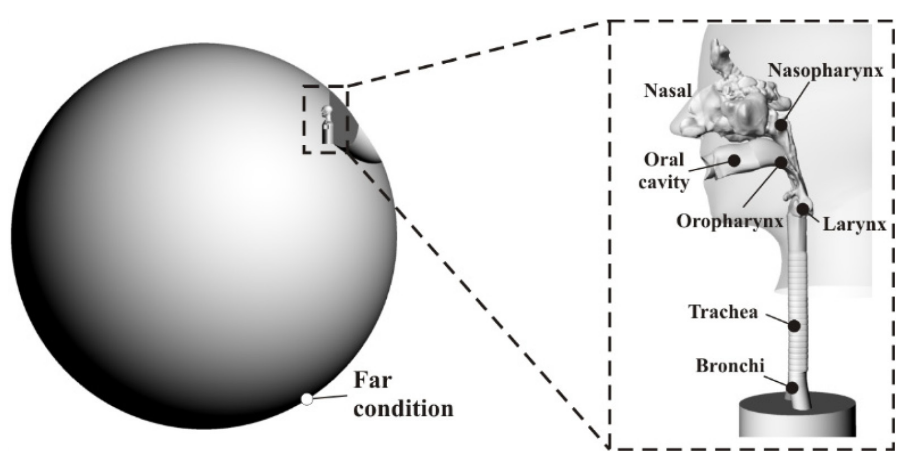

Figure 2: $\quad$ Model simulated.

With the geometry added, the final upper airway model comprised the mouth and nasal passages, in parallel with paranasal sinuses, the oral cavity, naso- and oro-pharynx, and larynx. The upper tracheobronchial region, including the trachea and the main bronchus bifurcation were also presented in the model. According to the standard nomenclature ICRP [15], the region created is a full extrathoracic model. The final geometry created is shown in detail in figure 2. Finally the complete model was imported into Gambit, the CFD pre-processor for meshing.

\section{Numerical modelling}

\subsection{Mesh}

The meshes were generated using Gambit and Tgrid, which are the preprocessing modules of the ANSYS. The convoluted and complex geometry of the scanned model greatly complicates the generation of a structured mesh; therefore a tetrahedral unstructured mesh was generated.

The completed model was divided into different volumes, cutting the airways with several cross sections. First, the superficial mesh was created in Gambit, with a constant value in main areas, and gradually increasing in size from the 
face to the far region. After that the boundary layer in airways was meshed with a growing prismatic cell layer in Tgrid. Finally, the core of all volumes, and the external region were meshed with an unstructured tetrahedral cell mesh.

In this work, in order to evaluate the mesh independence test, according to the CFD Best Practice Guidelines (Ansys [16]), the process was repeated six times. The results were six different mesh sizes: from $10^{5}$ cells in the coarsest mesh, to 25 million cells in the smoothest, with the first cell size always adjusted to attain a $y^{+}$value of approximately 1 .

Analysing the results of wall shear stress average on the walls at $30 \mathrm{~L} / \mathrm{min}$, the solution of compromise between accuracy and computational cost was the fourth mesh. The quality of the final mesh used in this paper is described in table 1 . Once the resulting mesh had passed all quality checks, the mesh was exported to Fluent, in which the boundary conditions, models, and materials were set up.

Table 1: $\quad$ Average mesh properties.

\begin{tabular}{|c|c|c|c|c|}
\hline Cell number & Cell skewness & Aspect ratio & Size change & Cell size \\
\hline 6615309 & 0.228 & 1.18 & 1.43 & $3.27 \cdot 10^{-5} \mathrm{~m}^{3}$ \\
\hline
\end{tabular}

\subsection{Fluid flow modelling}

The air flow through the extrathoracic model, and the surrounding mouth and nose area was simulated with the Ansys 14 software Fluent. The air was considered as pure air with properties at $20^{\circ} \mathrm{C}$, with no humidity or particles load. Analysing the flow rate range simulated and the airway dimensions, a low Mach number was verified in all conditions, so the air was considered as incompressible ideal gas. The viscosity was modelled following the three coefficients Sutherland method.

The airflow was solved using an Eulerian description, and segregated solver was adopted. The different analysed respiratory conditions and the variable geometry make it possible for the flow to change from a laminar to turbulent regime depending on the case or area studied. Therefore both laminar and turbulent RANS-based approaches were used. Previous studies in similar fields of airway simulation recommend the utilization of the $k-\omega$ model for modelling turbulent effects. Different studies $[2,17,18]$ demonstrate that this model is the best to capture the laminar to turbulent transition situations, and resolve the mean velocity distribution adequately. The enabling of the low Reynolds shear stress transport (SST) model is also recommended (Wilcox [19]).

The pressure-based solver was used with the SIMPLE pressure-velocity coupling scheme. Gradients were solved with the least squares cell based discretization and the standard model for pressure. Momentum and turbulence equations were solved with the high order QUICK scheme. 


\subsection{Boundary conditions}

Respiratory conditions from rest to extreme physical activity were simulated, with an air flow that ranged from 15 to more than $100 \mathrm{~L} / \mathrm{min}$. These simulated air flow rates were not imposed as boundary conditions; they were the result of the different pressure drops that were simulated.

Mass flow inlet, or uniform pressures are typically imposed in nostril and mouth in previous literature (Riazudiin et al. [20]), for simplicity. In this study a more realistic far condition was used. This was considered to be distant enough to maintain constant atmospheric pressure and null velocity, as we mention in the model paragraph. At the other extreme, the vacuum generator, the lungs were reproduced with a piston wall, enabling future unsteady simulations. Hence, pressure inlet and pressure outlet were imposed alternatively for inspiration and exhalation, on the far and piston walls. As result the air moves through the model according to the pressure difference imposed in each case.

All the walls of the extrathoracic model were assumed to be smooth, non-slip, and adiabatic. In this research mucous or other physiological aspects were not considered. In all the simulations the nasal and oral routes were totally open. No deformation, obstructions, or movements were considered.

\subsection{Convergence}

The converged solution of each case was reached following the same procedure under double precision. First the case was solved with first order scheme, until reaching convergence. The convergence criterion, following the fluent recommendations (Ansys [16]), was that all the residual of each variable fall below $10^{-3}$ at least. After first order scheme convergence, the case was changed to QUICK for all equations and iterated again until final convergence. In addition to the equation scaled residuals criterion, the convergence was judged by monitoring, the pressure drop, and the averaged and maximum wall shear stress.

The simulation was performed with Fluent solver, within the ANSYS workbench code on an Intel ${ }^{\circledR}$ Xeon $^{\circledR}$ Quad -Core E5530 2.4 GHz cluster with 156 GB of RAM.

\section{Results and discussion}

\subsection{Laminar vs k- $\omega$}

The model was simulated under laminar and turbulent conditions for inhalation and exhalation situation, and covering the full range of respiratory flow. The results are presented in figure 3 . The simulations were done with both routes, nasal and oral, open. So it is possible to plot the flow distribution through both routes during the inhalation and exhalation phases. In figure 3(a), the inhalation volumetric flow distribution under laminar and turbulent simulations is presented. The profiles obtained are very similar for the oral flow, and quite coincident for nasal flow. This shows that the inspiratory flow, with a low flow 
rate, is dominated by the laminar regimen. However the oral flow rate, higher than the nasal, has a different behaviour in the laminar than in the turbulent simulation, so the oral air flow in the inhalation phase is affected by turbulence effects. Similar results were found in the exhalation simulations (fig. 3(b)). The lower flow rates show the same behaviour in the laminar and turbulent simulations, and with higher flow values, on the right side of the figure, the curves separate clearly in an increasing tendency. In the exhalation phase the flow is more affected by the turbulent regimen in both routes at high volume flows.

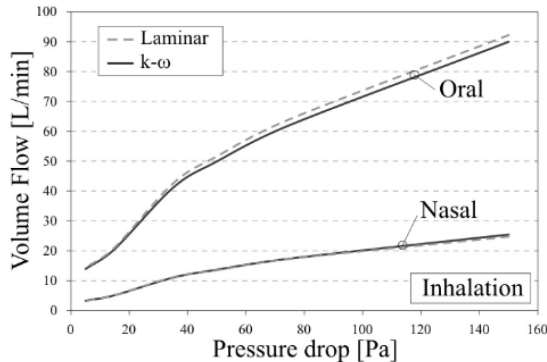

a

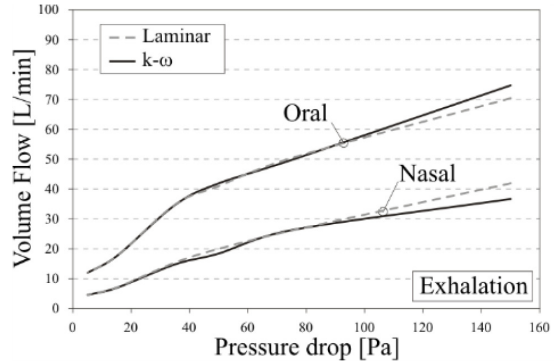

b

Figure 3: Laminar and turbulent volume flow distribution during (a) inhalation, (b) exhalation.

\subsection{Airflow analysis}

The extrathoracic model used is the result of joining scanned and model geometry. The complete model was simulated under simultaneous oral and nasal airflow rates under different respiratory conditions.

The different breathing conditions, corresponding to different activity levels, were achieved with different pressure drops from the lungs to the far away virtual surface at atmospheric pressure.

We are aware that simulated flow is not totally realistic due to changes of airway geometry that may occur during the respiration process, such as the movement of the palatal region. In any case it remains of interest to analyse the flow distribution through the two air flow routes. The oral route, with rates of around $75 \%$, is the major contributor to the amount of air breathed in the inhalation and exhalation phases. The contribution of these two passages to the total breathed volume for both phases is presented in figure 4 at different pressure drops. The rate obtained is not affected by the respiration level. The distribution is nearly constant for the full pressure drop range simulated, corresponding to the different breathing conditions.

Following a unique colour code, in figure 5 the volume flows at different pressure jumps are compared in pairs. The difference between oral and nasal flow in the inhalation phase, fig. 5(a) is clearly greater than in the exhalation phase, fig. 5(c). This indicates a more uniform flow resistance through both airways. The comparison from inhalation to exhalation by the same route, 


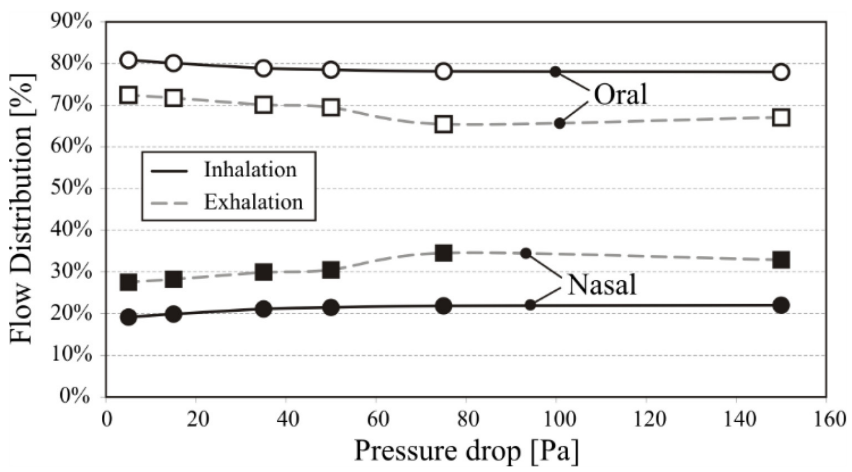

Figure 4: Volume flow distribution through oral and nasal routes in inhalation and exhalation.

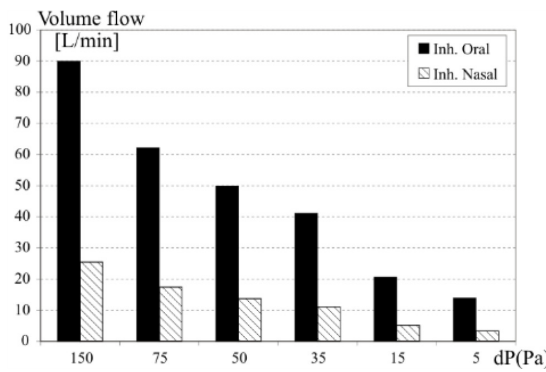

(a)

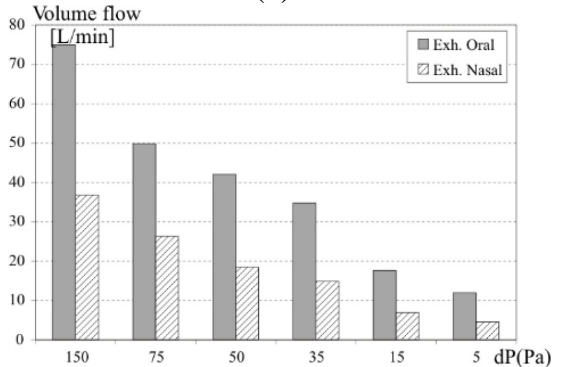

(c)

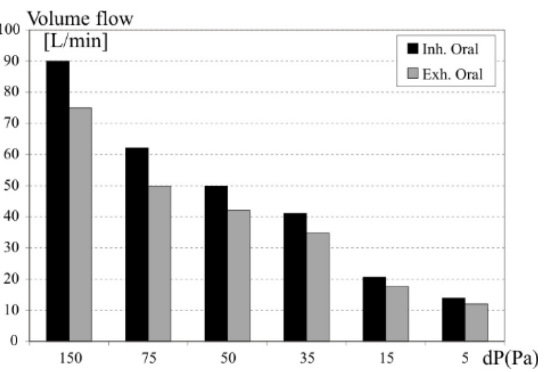

(b)

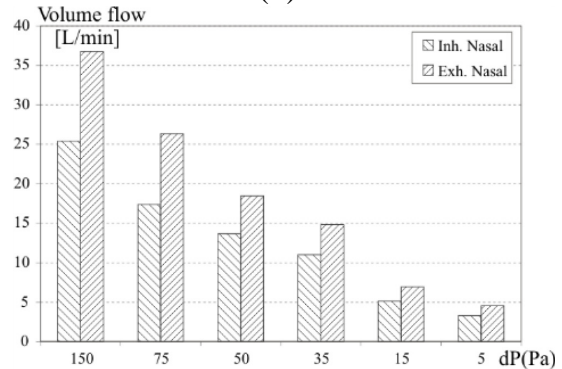

(d)

Figure 5: Comparative volume flow through the routes in each breathing phase.

figures 5(b) and 5(d), show the decrease of oral air flow, and the increase of nasal air flow to a greater extent.

The results obtained do not follow the expected behaviour. Under rest conditions most people inhale only through the nose (Bennett et al. [21]). Increasing physical activity the oral-nasal rate changes depending on the person (Malarbet et al. [22]), until reaching a high level of activity with total oral 
breathing. In the results obtained the oral airway was clearly presented as the preferred option for the air flow. Important differences appear between subjects depending on position, habits, or even racial differences, which could explain the specific results.
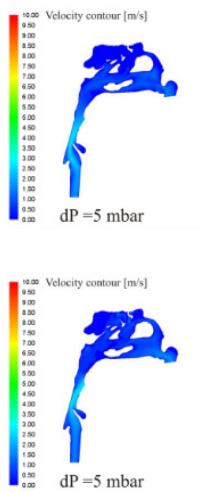
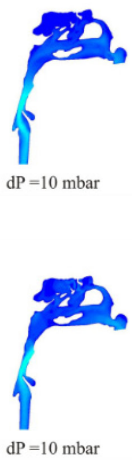
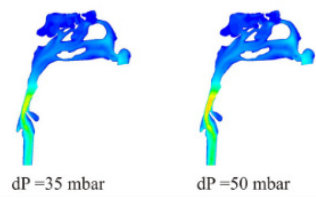

(a)

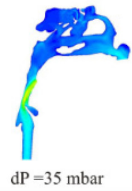

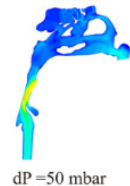

$\mathrm{dP}=50 \mathrm{mbar}$
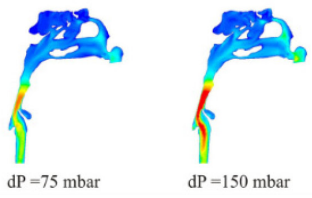

$\mathrm{dP}=150$ mbar

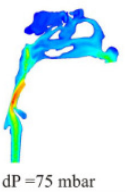

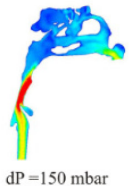

$\mathrm{dP}=150$ mbar

(b)

Figure 6: Sagittal section coloured by velocity (a) inhalation and (b) exhalation at different pressure drops.

The air flow effort changes from inhalation to exhalation, explaining the differences between figure 5(b) and 5(d). In figure 6, a sagittal plane coloured by velocity contour is presented for both respiratory phases. The flow distribution and the peak velocity areas are clearly different in each phase. The velocity contour results show a clear relationship with the concept of respiratory effort. Another interesting result is the almost negligible air flow through the nasal sinuses, in agreement with previous publications [2, 5, 9, 17, 18, 20].

\section{Conclusions}

A full extrathoracic airway geometry has been built as the sum of model and scanned data. The objective of obtaining a CFD model based on a CT scan has been successfully met.

Air flow patterns and flow distribution through oral and nasal routes were analysed and discussed under different breathing conditions. The flow behaviour and the respiratory effort changed notably from the inhalation to the exhalation phase, and are especially sensitive to the airway geometry of the individual.

The full process has been established and the steps and methodology have been standardized for future research. The results presented enhance the understanding of the breathing process. The realistic results indicate that this process is an appropriate analysis tool. This research constitutes a starting point toward a more ambitious aim of virtual surgery. 


\section{Acknowledgement}

Thanks to POVISA Hospital which has collaborated in the essential scan process.

\section{References}

[1] Ma, B., Ruwet, V., Corieri, P., Theunissen, R., Riethmuller, M. and Darquenne, C., CFD simulation and experimental validation of fluid flow and particle transport in a model of alveolated airways. Journal of Aerosol Science, 40, pp. 403-414, 2009.

[2] Mylavarapu, G., Murugappan, S., Mihaescu, M., Kalra, M., Khosla, S. and Gutmark, E., Validation of computational fluid dynamics methodology used for human upper airway flow simulations. Journal of Biomechanics, 42, pp. 1553-1559, 2009.

[3] Brouns, M., Verbanck, S. and Lacor, C., Influence of glottic aperture on the tracheal flow. Journal of Biomechanics, 40, pp. 165-172, 2007.

[4] Zhang, Z., Kleinstreuer, C. and Kim, C., Micro-particle transport and deposition in a human oral airway model. Journal of Aerosol Science, 33, pp. 1635-1652, 2002.

[5] Xi, J. and Longest, P.W., Transport and deposition of micro-aerosols in realistic and simplified models of the oral airway. Annals of Biomedical Engineering, 35, pp. 560-581, 2007.

[6] Jayaraju, S., Brouns, M., Verbanck, S. and Lacor, C., Fluid flow and particle deposition analysis in a realistic extrathoracic airway model using unstructured grids. Journal of Aerosol Science, 38, pp. 494-508, 2007.

[7] Saksono, P.H., Nithiarasu, P., Sazonov, I. and Yeo, S.Y., Computational flow studies in a subject-specific human upper airway using a one-equation turbulence model. Influence of the nasal cavity. International Journal for Numerical Methods in Engineering, 87, pp. 96-114, 2011.

[8] Inthavong, K., Choi, L.T., Tu, J., Ding, S. and Thien, F., Micron particle deposition in a tracheobronchial airway model under different breathing conditions. Medical Engineering and Physics, 32, pp. 1198-1212. 2010.

[9] Zhang, Z. and Kleinstreuer, C., Computational analysis of airflow and nanoparticle deposition in a combined nasal-oral-tracheobronchial airway model. Journal of Aerosol Science, 42, pp. 174-194, 2011.

[10] Kiraly, A.P., Higgins W.E., McLennan, G., Hoffman, E.A. and Reinhardt, J.M., Three-dimensional human airway segmentation methods for clinical virtual bronchoscopy. Academic Radiology, 9(10), pp. 1153-1168, 2002.

[11] Beare, R., A locally constrained watershed transform. IEEE Transactions on Pattern Analysis and Machine Intelligence, 28(7), pp. 1063-1074, 2006.

[12] Piegl, L.A. and Tiller, W., The NURBS book. New York, NY: SpringerVerlag, 1995.

[13] Johnstone, A., Uddin, M., Pollard, A., Heenan, A. and Finlay, W.H., The flow inside an idealised form of the human extra-thoracic airway. Experiments in Fluids, 37, pp. 673-689, 2004. 
[14] Malvè, M., Palomar, A.P.d., López-Villalobos, J., Ginel, A. and Doblaré, M., FSI analysis of the coughing mechanism in a human trachea. Annals of Biomedical Engineering, 38, pp. 1556-1565, 2010.

[15] ICRP, Human respiratory tract model for radiological protection. Annals of ICRP, 24, pp. 1-482, 1994.

[16] Ansys, Best Practices Guidelines for the CFD, 2006.

[17] Wen, J., Inthavong, K., Tian, Z.F., Tu, J.Y., Xue, C.L. and Li, C.G., Airflow Patterns in Both Sides of a Realistic Human Nasal Cavity for Laminar and Turbulent Conditions. 16th Australasian Fluid Mechanics Conference (AFMC), School of Engineering, The University of Queensland, Brisbane, Australia, pp. 68-74, 2007.

[18] Wen, J., Inthavong, K., Tu, J. and Wang, S, Numerical simulations for detailed airflow dynamics in a human nasal cavity. Respiratory Physiology and Neurobiology, 161, pp. 125-135, 2008.

[19] Wilcox, D.C., Turbulence Modeling for CFD DCW Industries, DCW Industries, Inc., 1998.

[20] Riazuddin, V.N., Zubair, M., Abdullah, M.Z., Ismail, R., Shuaib, I.L., Hamid, S.A. and Ahmad, K.A., Numerical study of inspiratory and expiratory flow in a human nasal cavity. Journal of Medical and Biological Engineering, 31, pp. 201-206, 2010.

[21] Bennett, W., Zeman, K. and Jarabek, A., Nasal contribution to breathing with exercise: effect of race and gender. Journal of Applied Physiology, 95, pp. 497-503, 2003.

[22] Malarbet, J.L., Bertholon, J.F., Becquemin, M.H., Taieb, G., Bouchikhi, A. and Roy, M., Oral and Nasal Flowrate Partitioning in Healthy Subjects Performing Graded Exercise. Radiation Protection Dosimetry, 53, pp. 179182, 1994. 